\title{
Desenvolvimento de solução IoT para monitoramento de gases
}

\author{
Leandro Augusto de Carvalho \\ Estudante do Programa de Pós-Gradua- \\ ção de Tecnologias Computacionais \\ para o Agronegócio \\ Medianeira, Paraná, Brasil \\ leandrocarvalho@utfpr.edu.br
}

\author{
Pedro Luiz de Paula Filho \\ Coordenador do Programa de Pós-Gra- \\ duação de Tecnologias Computacionais \\ para o Agronegócio \\ Medianeira, Paraná, Brasil \\ pedrol@utfpr.edu.br
}

\author{
Laércio Mantovani Frare \\ Professor do Programa de Pós-Gradua- \\ ção de Tecnologias Computacionais \\ para o Agronegócio \\ Medianeira, Paraná, Brasil \\ laercio@utfpr.edu.br
}

\begin{abstract}
The purpose of this article is to detail the development of an IoT solution for monitoring gases. The solution consists of a prototype responsible for measuring ammonia and carbon dioxide gases, using the MQ-137 and MG-811 sensors, respectively. The collected data is processed by an ESP-32 prototyping card, and sent over a wireless network, to a data server. This server is responsible for storing and displaying information on the ThingsBoard platform. To evaluate the solution, the monitoring of the environmental conditions of a poultry breeding unit was carried out over a period of four days. The results obtained show that the prototype was able to perform gas measurements during the testing period.
\end{abstract}

Resumo - O objetivo desse trabalho consiste em detalhar o desenvolvimento de uma solução IoT para monitoramento de gases. A solução consiste em um protótipo responsável pela medição dos gases amônia e dióxido de carbono, utilizando os sensores MQ-137 e MG-811, respectivamente. Os dados coletados são processados por uma placa de prototipagem ESP-32, e enviados por meio de rede sem fio, para um servidor de dados. Este servidor é responsável por armazenar e apresentar as informações na plataforma ThingsBoard. Para avaliar a solução, foi realizado o monitoramento das condições ambientais de uma unidade de criação de aves, no período de quatro dias. Os resultados obtidos mostram que o protótipo foi capaz de realizar as medições dos gases durante o período de testes.

Palavras-chave-monitoramento; gases; protótipo.

\section{INTRODUÇÃO}

No Brasil, a Norma Regulamentadora $\mathrm{n}^{\circ} 15$, ou NR-15 como é mais conhecida, refere-se as condições de trabalho consideradas insalubres. Essa norma trata desde as questões relacionadas a ergonomia, até a saúde e a segurança dos trabalhadores. Segundo essa norma a qualidade do ar deverá ser mantida dentro dos padrões de pureza estabelecidos, e nas atividades ou operações nas quais os trabalhadores ficam expostos a agentes químicos, a caracterização de insalubridade ocorrerá quando forem ultrapassados os limites de tolerância fixados [1].

Ainda no que diz respeito a segurança e saúde dos trabalhadores, há outras normativas como a NR-33, que refere-se a identificação de espaços confinados e o reconhecimento, avaliação, monitoramento e controle dos riscos existentes. Parte das medidas técnicas de prevenção consistem em avaliar a atmosfera nos espaços confinados antes da entrada de trabalhadores, e monitorar continuamente a atmosfera nas áreas onde os trabalhadores estiverem desempenhando as suas tarefas [2].

Essas condições de insalubridade podem ser encontradas em diversos ramos de atividade como nas granjas de criação de frango de corte, onde ocorre a formação e acúmulo de gases como amônia $\left(\mathrm{NH}_{3}\right)$ [3] e dióxido de carbono $\left(\mathrm{CO}_{2}\right)$ [4], e também em instalações suinícolas [5].

Sendo assim, as atividades que envolvem trabalhadores expostos a gases nocivos a saúde, ou então a qualidade do ar impróprias, estão sujeitas às normativas vigentes, e portanto, são passíveis de monitoramento e controle. Geralmente são utilizados equipamentos detectores portáteis para realizar a mensuração dos níveis dos gases nos ambientes, porém, esses equipamentos podem representar um alto custo, além de não fornecer o monitoramento ativo das condições ambientais em tempo real.

Uma forma de realizar esse monitoramento consiste na implantação de equipamentos sensores nos ambientes onde ocorre o acúmulo dos gases nocivos, de modo que as leituras desses sensores possam ser transmitidas para um servidor de dados, responsável por apresentar as condições ambientais e fornecer as ferramentas de apoio para o controle dessas condições.

É nesse contexto que os conceitos de Internet das Coisas (IoT) se integram com o desenvolvimento da solução. $\mathrm{O}$ termo IoT pode ser definido como a rede ubíqua e global que ajuda e provê a funcionalidade de integrar o mundo físico. Isso se dá por meio da coleta, do processamento e da análise de dados gerados pelos sensores da IoT [6].

Portanto, o presente trabalho visa apresentar o desenvolvimento de uma solução de baixo custo para monitoramento de gases de forma contínua, utilizando soluções de código aberto, desde a construção do protótipo até apresentação dos dados em plataforma IoT. Para avaliar o desempenho do protótipo, foram realizados testes em aviário de criação de frango de corte, na cidade de Cascavel-PR, monitorando os níveis de dióxido de carbono $\left(\mathrm{CO}_{2}\right)$ e amônia $\left(\mathrm{NH}_{3}\right)$ no interior do galpão. 


\section{COMPONENTES DA SOLUÇÃO}

Para esse protótipo foram utilizados sensores semicondutores genéricos, modelo MQ-137 para detecção do gás amônia, e MG-811 para detecção do gás dióxido de carbono. Esses sensores foram escolhidos pelo seu baixo custo em relação a outros tipos de sensores como os eletroquímicos, por exemplo, e por serem facilmente encontrados para aquisição.

O sensor MQ-137 opera na faixa de detecção de 5 até 200 partes por milhão (ppm) de amônia, em temperatura ambiente de $20{ }^{\circ} \mathrm{C}$ e umidade relativa do ar de $65 \%$ [7]. É compatível com diversas placas de prototipagem o que traz versatilidade para o componente, apresentado na figura 1 .

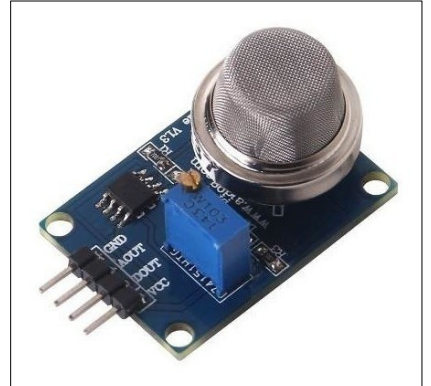

Fig. 1. Sensor de Amônia MQ-137

O sensor MG-811, exibido na figura 2, trabalha na faixa de detecção de dióxido de carbono, entre 350 e 10.000 ppm, em temperatura ambiente de $28^{\circ} \mathrm{C}$ e umidade relativa do ar em $65 \%$ [7].

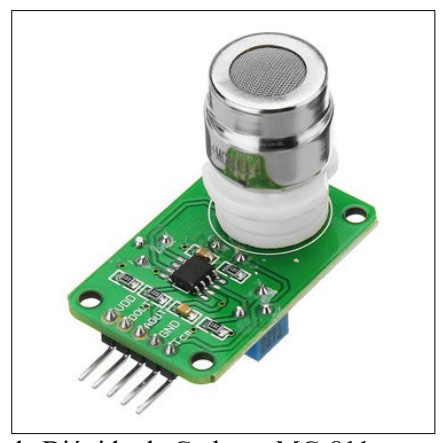

Fig. 2. Sensor de Dióxido de Carbono MG-811

Ambos sensores utilizados são do tipo semicondutores, de óxido metálico. O funcionamento desse tipo de sensor baseia-se na interação do gás alvo com a superfície do filme óxido metálico, resultando em uma mudança na concentração do portador de carga do material, alterando sua condutividade ou resistividade [9].

A alteração na condutividade é o valor detectado pela placa de prototipagem e convertido, utilizando uma equação de calibração, em unidade de medida de concentração. A equação de calibração é diferente para cada tipo de sensor, porém, ambas são extraídas dos gráficos de sensibilidade apresentados no datasheet dos sensores.

A placa de prototipagem escolhida para o desenvolvimento do protótipo é conhecida como ESP32 ou DevKit ESP32, produzida pela empresa Espressif Systems. As principais características desta placa são chip com WiFi embutido padrão $802.11 \mathrm{~b} / \mathrm{g} / \mathrm{n}$, operando na faixa de 2.4 a $5 \mathrm{GHz}$, modos de operação cliente, ponto de acesso e cliente+ponto de acesso, conexão Bluetooth versão 4.2, módulo controlador ESP WROOM 32, microprocessador dual core Tensilica Xtensa 32 bit LX6, clock ajustável de $80 \mathrm{MHz}$ até $240 \mathrm{MHz}, 36$ portas programáveis de entrada e saída [10].

Para realizar uma cópia de segurança e evitar perda de dados, um módulo de cartão microSD foi incorporado ao protótipo, realizando o registro das leituras em arquivo de texto no cartão de memória. Esse módulo é genérico, ou seja, não possui marca ou indicação do fabricante, tem preço acessível, e utiliza comunicação serial síncrona no protocolo Serial Peripheral Interface (SPI).

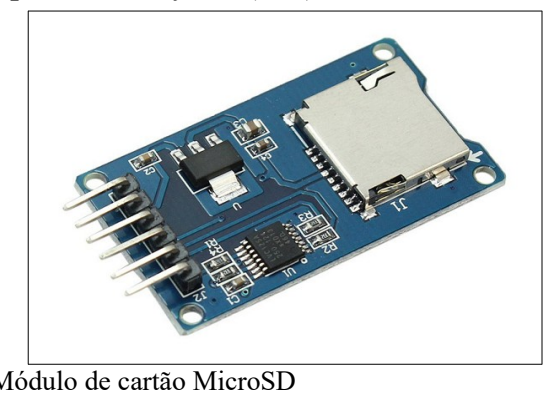

Fig. 3. Módulo de cartão MicroSD

Para auxiliar na montagem e manutenção dos protótipos, uma placa de circuito impresso foi desenvolvida utilizando placa de fenolite de face simples e barras de pinos fêmea 180 graus, permitindo que os cabos jumper fossem facilmente conectados entre os componentes (sensores e módulo de cartão microSD). Alguns pinos extras foram destinados para conexão de outros sensores como sensor de temperatura e umidade, para projetos futuros. A figura 4 apresenta $o$ desenho do circuito feito no software Fritzing[11].



Fig. 4. Placa de circuito impresso

Para finalizar o protótipo, uma caixa de montagem foi utilizada para acomodar todos os componentes que não necessitavam ficar expostos (placa de prototipagem e módulo microSD) e a tampa da caixa foi perfurada permitindo a exposição dos sensores ao ambiente. Como os testes foram realizados em um aviário, uma tela de proteção foi instalada cobrindo a parte frontal da caixa, evitando o acúmulo de poeira nos sensores.

Como o objetivo era realizar o monitoramento contínuo dos gases, foi necessário agregar a solução, um sistema capaz de armazenar e apresentar os dados coletados chamado ThingsBoard. Esse sistema é uma plataforma IoT de código aberto para coleta de dados, processamento, visualização e gerenciamento de dispositivos [12]. 
Esse sistema foi implantado localmente, instalado em um computador de placa única Raspberry PI 3 Modelo B, em ambiente RaspbianOS, representando o servidor de dados dentro da solução.

\section{FUNCIONAMENTO}

A comunicação entre o protótipo e o servidor de dados realizou-se por meio de rede sem fio, utilizando um ponto de acesso e a configuração de rede necessária nos equipamentos. $\mathrm{O}$ protocolo utilizado para troca de mensagens foi o Message Queuing Telemetry Transport (MQTT), compatível com a plataforma ThingsBoard.

Esse protocolo de transporte de mensagens opera com o conceito de publicação e assinatura entre o cliente e servidor. Caracteriza-se por ser um protocolo leve, aberto, simples e projetado para fácil implementação. Ideal para uso em ambientes restritos, como para comunicação em Machine to Machine (M2M) e IoT [13].

A figura 5 apresenta um esquema da comunicação entre os componentes.

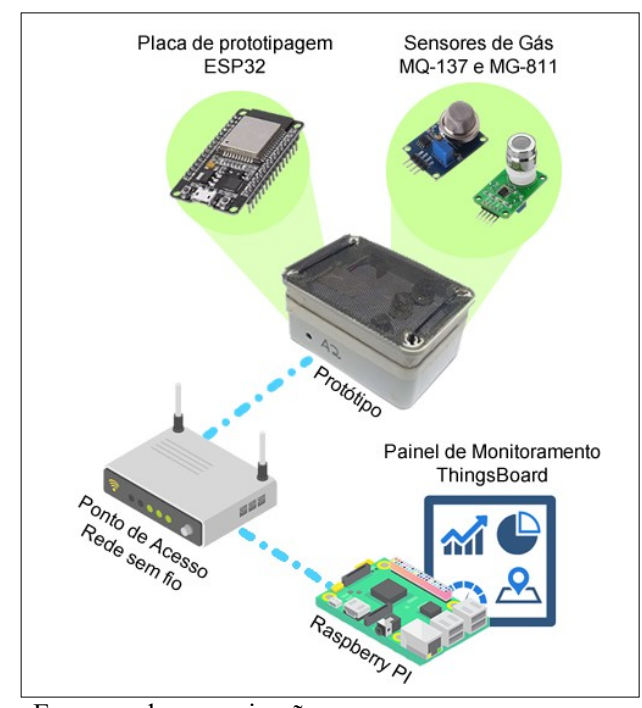

Fig. 5. Esquema de comunicação

Quanto a programação da placa de prototipagem, foram criadas as rotina de coleta e tratamento dos dados, conexão na rede sem fio, conexão com o servidor ThingsBoard, e envio dos dados.

Como a coleta dos dados foi programada para acontecer uma vez a cada trinta segundos, o início da rotina acontecia de forma agendada. A figura 5 apresenta o diagrama de atividades das rotinas.

Após a coleta dos dados, as leituras passam por uma conversão, transformado o valor lido pelo sensor na porta analógica para um valor na unidade ppm do referido gás, e são registrados no arquivo de $\log$.

Feito isso, a próxima etapa consiste em conexão na rede sem fio com as credenciais pré-definidas e, após, conexão com o serviço MQTT oferecido pelo servidor ThingsBoard. A conexão com o servidor também requer autenticação, uma vez que o dispositivo que encaminhará as mensagens deve estar inserido no sistema.

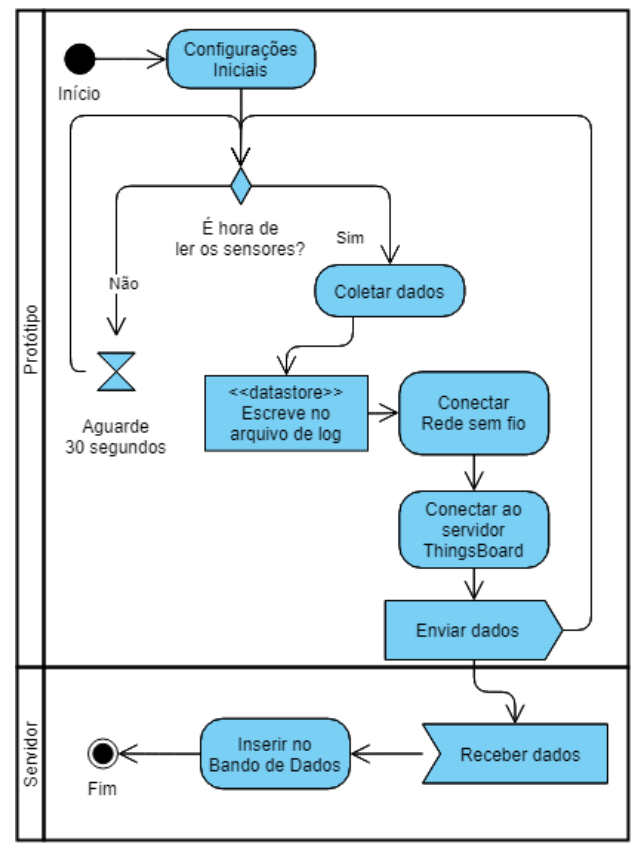

Fig. 6. Diagrama da atividade principal

Para que as mensagens encaminhadas para o servidor possam ser inseridas no banco de dados e exibidas nos painéis de monitoramento, é necessária uma etapa de codificação no formato JavaScript Object Notation (JSON). JSON é um formato de intercâmbio de dados independente da linguagem de programação, leve, e baseado em texto. Esse formato funciona, basicamente, no padrão atributovalor, onde, nesse caso, os atributos devem ser os campos inseridos previamente no sistema ThingsBoard, e o valor é referente a leituras dos sensores [14].

Ao receber os dados enviados pelo protótipo, o sistema realiza a validação dos atributos e insere no banco de dados. Para recuperar os valores das leituras, pode-se consultar diretamente o banco de dados, ou então, criar painéis de monitoramento no sistema ThingsBoard, de modo a permitir a exportação desses dados. Esses painéis podem conter elementos gráficos como séries de dados temporais, indicadores, mapas, entre outros componentes visuais para facilitar a apresentação.

A figura 7 apresenta o painel de monitoramento criado para o acompanhamento das condições em tempo real, acessado pelo navegador de internet do servidor.

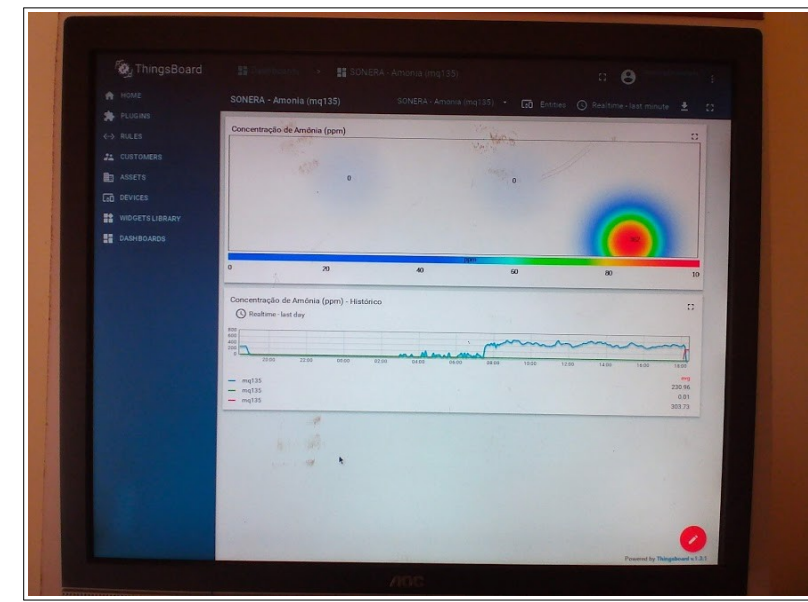

Fig. 7. Painel de monitoramento 
Para avaliar o funcionamento da solução, um aviário de criação de frango de corte, localizado no município de Cascavel-PR, foi utilizado para instalação do protótipo e do servidor de monitoramento.

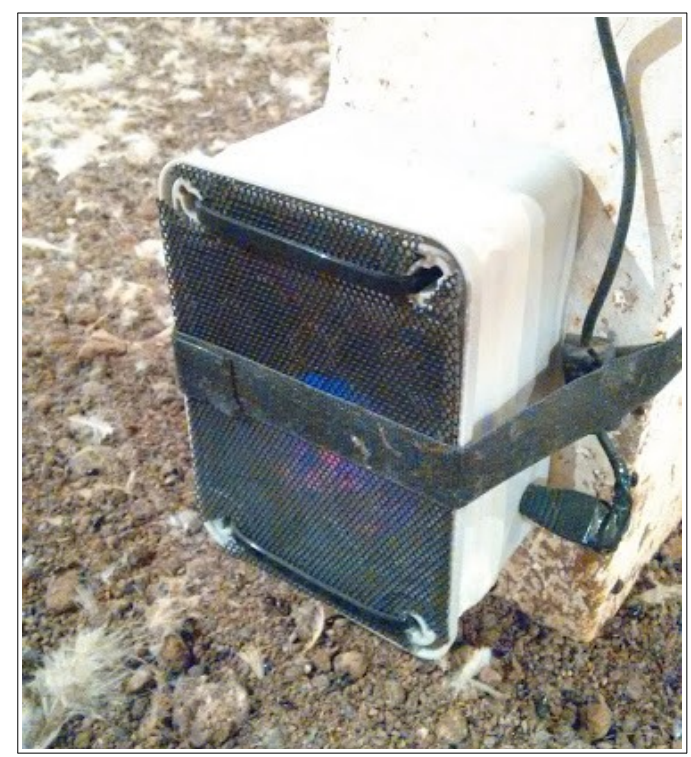

Fig. 8.

Protótipo instalado no aviário

O aviário possui dimensões de $12 \mathrm{~m}$ de largura por $100 \mathrm{~m}$ de comprimento, totalizando uma área de $1.200 \mathrm{~m}^{2}$, com pédireito de 2,2 metros e pilares de concreto a cada 3 metros, com tesouras de madeiras e cobertura de telhas de barro. O protótipo foi instalado em um dos pilares de sustentação do galpão, com os sensores em posição perpendicular ao solo. $\mathrm{O}$ protótipo permaneceu junto ao solo, porém, os sensores encontravam-se a uma altura de $15 \mathrm{~cm}$ do chão. Para a análise dos resultados, o protótipo permaneceu coletando dados durante todo o período dos dias $2,4,5$ e 8 de abril de 2019.

\section{RESULTADOS E DISCUSSÃO}

Como as coletas eram realizadas a cada trinta segundos, durante um dia inteiro de coleta haveriam 2.880 registros no banco de dados por sensor. Para facilitar a análise e representação, os dados foram agrupados a cada quinze minutos amostrais, utilizando a média das leituras.

Houveram diferenças na quantidade de coletas realizadas pelo protótipo entre os dias estudados, devido a falhas momentâneas no fornecimento de energia elétrica. Porém, nos dias em que ocorreram as falhas, a solução retornou ao pleno funcionamento após os incidentes serem resolvidos, sem a necessidade de intervenção no protótipo ou no servidor de dados.

A Tabela 1 apresenta a quantidade de registros agrupados por dia amostral.

TABELA I

QUANTIDADE DE REGISTROS POR DIA

\begin{tabular}{|c|c|c|c|c|}
\hline & $\mathbf{0 2 / 0 4 / 1 9}$ & $\mathbf{0 4 / 0 4 / 1 9}$ & $\mathbf{0 5 / 0 4 / 1 9}$ & $\mathbf{0 8} / \mathbf{0 4} / \mathbf{1 9}$ \\
\hline $\begin{array}{c}\text { Grupos de } \\
\text { registro }\end{array}$ & 69 & 67 & 96 & 92 \\
$(71,87 \%)$ & $(69,79 \%)$ & $(100 \%)$ & $(95,83 \%)$ \\
\hline
\end{tabular}

Apenas o dia 5 obteve o registro de todas as leituras, e portanto, apresentou 96 grupos amostrais. Considerando o dia que obteve o menor número de leituras com sucesso, dia 4, com 67 agrupamentos, é possível afirmar que aproximadamente $69,8 \%$ das leituras possíveis em um dia foram bemsucedidas.

Referente aos valores obtidos pelos sensores, a figura 9 apresenta as leituras do sensor MQ-137, para os níveis de amônia, nos dias amostrais.

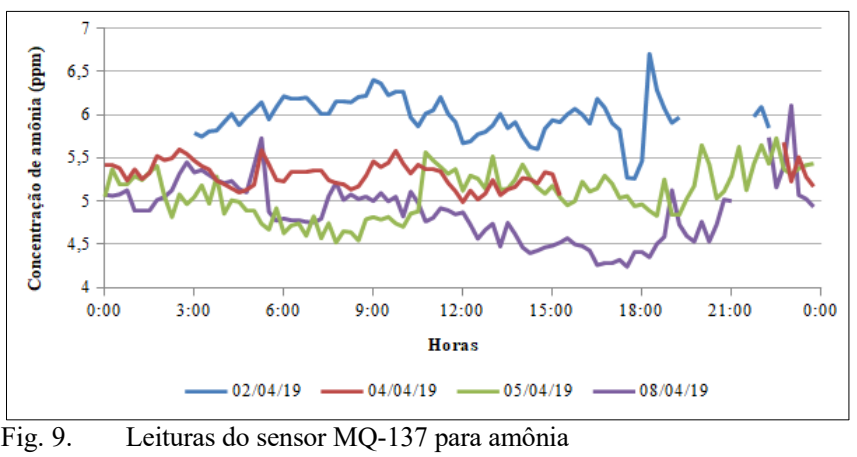

Como mostrado na Tabela 1, o número de amostras foi menor em alguns dias em relação aos demais. Realizando a análise visual do gráfico é possível concluir que ocorreram variações das concentrações de amônia entre os dias amostrais, e também ao longo das horas de cada dia.

Todas as leituras permaneceram dentro da faixa de 4 a 7 ppm, embora a folha de dados do sensor indique que a faixa de operação é de 5 a 200 ppm. Algumas hipóteses prováveis para essa situação são que o sensor não tenha precisão rigorosa, ou que podem haver erros na conversão dos valores.

Como todos os valores permaneceram abaixo de $20 \mathrm{ppm}$, as atividades no ambiente de teste estão dentro do limite de tolerância para exposição à amônia, segundo a NR-15 [1].

Os valores registrados para as concentrações de dióxido de carbono são apresentados na figura 10. Todas as leituras ficaram entre 315 e 335 ppm.

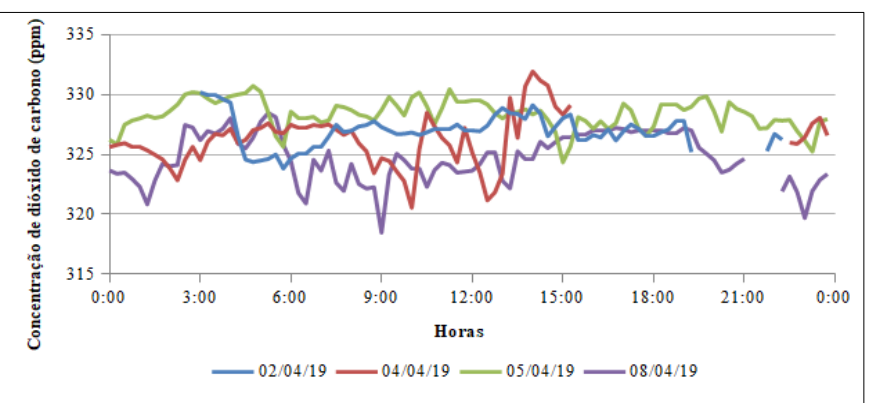

Fig. 10. Leituras do sensor MG-811 para dióxido de carbono

Sendo assim, não houve momentos onde a concentração excedeu o limite tolerável de $3.900 \mathrm{ppm}$, considerado insalubre segundo a NR-15 [1].

Um fato observado para ambos os sensores foi a necessidade de calibração utilizando um método certificado para mitigar os erros de conversão, ou então, utilizar um equipamento calibrado para realizar a comparação com as leituras registradas. Porém, nesse caso o ideal é que o equipamento certificado possua o mesmo tipo de sensor, uma vez que existem diferenças em tempo de resposta, faixa de operação entre outras características, que podem influenciar na comparação. 
Por fim, a solução mostrou-se bastante estável uma vez que não foi necessária nenhuma intervenção nos componentes, no período de testes.

\section{CONCLUSÃO}

O objetivo principal desse trabalho foi apresentar o desenvolvimento de uma solução IoT para monitoramento de gases, e, utilizando um aviário comercial de frango de corte como cenário de testes, validar os principais resultados obtidos.

Os sensores foram capazes de detectar as concentrações dos dois gases alvo sendo amônia e dióxido de carbono, em diferentes níveis de concentração.

A plataforma ThingsBoard não apresentou problemas no período de testes, mostrando-se uma eficiente alternativa para coleta e visualização de dados IoT.

Considerando que o aviário de testes não possuía nenhum tipo de equipamento para monitoramento de gases, essa solução torna-se uma alternativa viável para implantação, uma vez que não representa um alto custo para o produtor.

\section{REFERÊNCIAS}

[1] BRASIL. Ministério do Trabalho e Emprego. NR 15 - Atividade e operações insalubres. Brasília, 1978. Acesso em: 14 nov. 2020. [Online] Disponível em: https://enit.trabalho.gov.br/portal/images/Arquivos_SST/SST_NR/NR15-atualizada-2019.pdf

[2] BRASIL. Ministério do Trabalho e Emprego. NR-33 segurança e saúde nos trabalhos em espaços confinados. Brasília, 2006. Acesso em: 14 nov. 2020 [Online] Disponível em: https://enit.trabalho.gov.br/portal/images/Arquivos_SST/SST_NR/NR33.pdf

[3] P. G. Abreu, A. Coldebella, V. Feddern e J. I. Santos Filho, "Amônia em aviário dark house," in Congresso Nordestino De Produção Animal, Ilhéus, 2014. pp. 1032-1038. Acesso em: 14 nov. 2020. [Online] Disponível em: https://www.embrapa.br/busca-depublicacoes/-/publicacao/1013875/amonia-em-aviario-dark-house.
[4] I. Menegali, I. F. F. Tinôco, F. C. Baêta, P. R. Cecon, M. C. C. Guimarães e M. B. Cordeiro, "Ambiente térmico e concentração de gases em instalações para frangos de corte no período de aquecimento," Revista Brasileira de Engenharia Agrícola e Ambiental, vol. 13, supl. p. 984-990, Dez. 2009, doi: 10.1590/S141543662009000700022

[5] J. A. Campos, I. F. F. Tinôco, F. C. Baêta, P. R. Cecon e A. L. Mauri, "Qualidade do ar, ambiente térmico e desempenho de suínos criados em creches com dimensões diferentes”, Engenharia Agrícola, vol.29, n.3, p. 339-347, Set. 2009, doi: 10.1590/S0100-69162009000300001

[6] A. L. Albertin e R. M. M. Albertin, “A Internet das Coisas irá Muito Além das Coisas," GV-executivo, vol. 16, n. 2, p.12-17, Mar.-Abr. 2017. Acesso em: 14 nov. 2020. [Online] Disponível em: https://rae.fgv.br/sites/rae.fgv.br/files/gv_v16n2_ce1.pdf.

[7] Technical Data MQ-137 Gas Sensor. Acesso em: 14 nov. 2020; [Online]. Disponível em: https://datasheetspdf.com/pdf-file/904648/HANWEIELECTRONICS/ MQ-137/1

[8] Technical Data MG-811 Gas Sensor. Acesso em: 14 nov. 2020; [Online]. Disponível em: https://datasheetspdf.com/pdf-file/576123/ETC/MG811/1

[9] G. F. Fine, L. M. Cavanagh, A. Afonja, R. Binions, "Metal Oxide Semi Conductor Gas Sensors in Environmental Monitoring”. Sensors, vol. 10, n. 6, p. 5469-5502, 1 Jun. 2010, doi: 10.3390/s100605469.

[10] ESP32-WROOM-32D \& ESP32-WROOM-32U Datasheet v 1.9 (2019) Acesso em: 14 nov. 2020. [Online] Disponível em: https://www.espressif.com/sites/default/files/documentation/esp32wroom-32d_esp32-wroom-32u_datasheet_en.pdf

[11] "Fritzing" https://fritzing.org (acesso em $01 \mathrm{dez} .2020$ )

[12] “ThingsBoard - Open-Source IoT Platform" https://thingsboard.io (acesso em 14 nov. 2020)

[13] MQTT Version 5.0, OASIS Standard, Mar. 2019. [Online]. Disponível em: https://docs.oasis-open.org/mqtt/mqtt/v5.0/mqtt-v5.0.html

[14] The JavaScript Object Notation (JSON) Data Interchange Format, Dez. 2017. [Online]. Disponível em: https://tools.ietf.org/html/rfc8259 